\title{
NAKAI-MOISHEZON AMPLENESS CRITERION FOR REAL LINE BUNDLES
}

\author{
OSAMU FUJINO AND KEISUKE MIYAMOTO
}

\begin{abstract}
We show that the Nakai-Moishezon ampleness criterion holds for real line bundles on complete schemes. As applications, we treat the relative Nakai-Moishezon ampleness criterion for real line bundles and the Nakai-Moishezon ampleness criterion for real line bundles on complete algebraic spaces. The main ingredient of this paper is Birkar's characterization of augmented base loci of real divisors on projective schemes.
\end{abstract}

\section{Contents}

1. Introduction

2. Preliminaries 2

3. Augmented base loci of $\mathbb{R}$-divisors 3

4. Proof of Theorem 1.4 4

5. Proof of Theorem $1.3 \quad 5$

6. Proof of Theorem 1.5 7

7. Proof of Theorem $1.6 \quad 8$

References $\quad 9$

\section{INTRODUCTION}

Throughout this paper, a scheme means a separated scheme of finite type over an algebraically closed field $k$ of any characteristic. We call such a scheme a variety if it is reduced and irreducible. Let us start with the definition of $\mathbb{R}$-line bundles.

Definition 1.1 ( $\mathbb{R}$-line bundles). Let $X$ be a scheme (or an algebraic space). An $\mathbb{R}$-line bundle (resp. a $\mathbb{Q}$-line bundle) is an element of $\operatorname{Pic}(X) \otimes_{\mathbb{Z}} \mathbb{R}\left(\operatorname{resp}\right.$. $\left.\operatorname{Pic}(X) \otimes_{\mathbb{Z}} \mathbb{Q}\right)$ where $\operatorname{Pic}(X)$ is the Picard group of $X$.

Similarly, we can define $\mathbb{R}$-Cartier divisors.

Definition 1.2 ( $\mathbb{R}$-Cartier divisors). Let $X$ be a scheme. An $\mathbb{R}$-Cartier divisor (resp. a $\mathbb{Q}$-Cartier divisor $)$ is an element of $\operatorname{Div}(X) \otimes_{\mathbb{Z}} \mathbb{R}\left(\operatorname{resp} . \operatorname{Div}(X) \otimes_{\mathbb{Z}} \mathbb{Q}\right)$ where $\operatorname{Div}(X)$ denotes the group of Cartier divisors on $X$.

We prove the Nakai-Moishezon ampleness criterion for $\mathbb{R}$-line bundles. The following theorem is the main result of this paper.

Theorem 1.3 (Nakai-Moishezon ampleness criterion for real line bundles on complete schemes). Let $X$ be a complete scheme over an algebraically closed field and let $\mathcal{L}$ be an $\mathbb{R}$-line bundle on $X$. Then $\mathcal{L}$ is ample if and only if $\mathcal{L}^{\operatorname{dim} Z} \cdot Z>0$ for every positivedimensional closed integral subscheme $Z \subset X$.

Date: $2020 / 11 / 29$, version 0.09 .

2010 Mathematics Subject Classification. Primary 14C20; Secondary 14E30.

Key words and phrases. Nakai-Moishezon ampleness criterion, Kleiman's ampleness criterion, $\mathbb{R}$-line bundles, $\mathbb{R}$-Cartier divisors, augmented base loci, projectivity, algebraic spaces. 
When $X$ is projective, Theorem 1.3 is well known. It was first proved by Campana and Peternell (see [CP, 1.3. Theorem]). Then a somewhat simpler proof was given by Lazarsfeld in [La, Theorem 2.3.18]. Unfortunately, their arguments do not work for complete nonprojective schemes because they need an ample line bundle. Moreover, Kleiman's ampleness criterion does not always hold for complete nonprojective schemes (see [F1, Section 3] and [F4, Example 12.1]). Hence we need some new idea to prove Theorem 1.3. By the standard reduction argument, it is sufficient to treat the case where $X$ is a complete normal variety. Therefore, all we have to do is to establish the following theorem.

Theorem 1.4 (Nakai-Moishezon ampleness criterion for real Cartier divisors on complete normal varieties). Let $X$ be a complete normal variety over an algebraically closed field and let $L$ be an $\mathbb{R}$-Cartier divisor on $X$. Then $L$ is ample if and only if $L^{\operatorname{dim} Z} \cdot Z>0$ for every positive-dimensional closed subvariety $Z \subset X$.

For the proof of Theorem 1.4, we use Birkar's characterization of augmented base loci of $\mathbb{R}$-divisors on projective schemes (see Theorem 3.4). Hence our approach is different from those of $[\mathrm{CP}]$ and $[\mathrm{La}]$. Although we can not directly apply geometric arguments to $\mathbb{R}$-line bundles, we can generalize Theorem 1.3 for proper morphisms.

Theorem 1.5 (Relative Nakai-Moishezon ampleness criterion for real line bundles). Let $\pi: X \rightarrow S$ be a proper morphism between schemes and let $\mathcal{L}$ be an $\mathbb{R}$-line bundle on $X$. Then $\mathcal{L}$ is $\pi$-ample if and only if $\mathcal{L}^{\operatorname{dim} Z} \cdot Z>0$ for every positive-dimensional closed integral subscheme $Z \subset X$ such that $\pi(Z)$ is a point.

For the details of the Nakai-Moishezon ampleness criterion and Kleiman's ampleness criterion for line bundles, see $[\mathrm{Kl}]$. The reader can find many nontrivial examples of complete nonprojective varieties in [F1], [F4, Section 12], [FP], and so on. Finally, we prove the following theorem as an application of Theorem 1.3 by using some basic properties of algebraic spaces.

Theorem 1.6 (Nakai-Moishezon ampleness criterion for real line bundles on complete algebraic spaces). Let $X$ be a complete algebraic space over an algebraically closed field and let $\mathcal{L}$ be an $\mathbb{R}$-line bundle on $X$. Then $\mathcal{L}$ is ample if and only if $\mathcal{L}^{\operatorname{dim} Z} \cdot Z>0$ for every positive-dimensional closed integral subspace $Z \subset X$.

We note that we treat algebraic spaces only in the final section, where we prove Theorem 1.6. We also note that the Nakai-Moishezon ampleness criterion for line bundles on complete algebraic spaces plays an crucial role in Kollár's projectivity criterion for moduli spaces (see [Ko] and [F3]).

Acknowledgments. The first author was partially supported by JSPS KAKENHI Grant Numbers JP16H03925, JP16H06337. The second author was partially supported by JSPS KAKENHI Grant Number 20J20070. The authors thank Yoshinori Gongyo and Kenta Hashizume for comments.

\section{Preliminaries}

For simplicity of notation, we write the group law of $\operatorname{Pic}(X) \otimes_{\mathbb{Z}} \mathbb{R}$ additively.

Definition 2.1. Let $\mathcal{L}$ be an $\mathbb{R}$-line bundle on a complete scheme $X$.

- If $\mathcal{L}=\sum_{j} l_{j} \mathcal{L}_{j}$ such that $l_{j}$ is a positive real number and $\mathcal{L}_{j}$ is an ample line bundle on $X$ for every $j$, then we say that $\mathcal{L}$ is ample.

- If $\mathcal{L}=\sum_{j} l_{j} \mathcal{L}_{j}$ such that $l_{j}$ is a positive real number and $\mathcal{L}_{j}$ is a semi-ample line bundle on $X$ for every $j$, then we say that $\mathcal{L}$ is semi-ample.

- If $\mathcal{L} \cdot C \geq 0$ for every curve $C$ on $X$, then we say that $\mathcal{L}$ is nef. 
- We further assume that $X$ is a variety. If $\mathcal{L}=\sum_{j} l_{j} \mathcal{L}_{j}$ such that $l_{j}$ is a positive real number and $\mathcal{L}_{j}$ is a big line bundle on $X$ for every $j$, then we say that $\mathcal{L}$ is big.

In the theory of minimal models, we usually use $\mathbb{R}$-Cartier divisors. In this paper, we do not use $\mathbb{R}$-Weil divisors. We only use $\mathbb{R}$-Cartier divisors.

Definition 2.2. Let $X$ be a complete scheme. We consider the following natural homomorphism

$$
\psi: \operatorname{Div}(X) \otimes_{\mathbb{Z}} \mathbb{R} \rightarrow \operatorname{Pic}(X) \otimes_{\mathbb{Z}} \mathbb{R} .
$$

We note that $\psi$ is not necessarily surjective. Let $D$ be an $\mathbb{R}$-Cartier divisor on $X$. If the image of $D$ by $\psi$ is ample, semi-ample, nef, and big, then $D$ is said to be ample, semi-ample, $n e f$, and big, respectively. We also note that $\psi$ is surjective when $X$ is a variety.

For the basic properties of bigness and semi-ampleness, see [F2, Section 2.1]. Here we only explain the following important characterization of nef and big $\mathbb{R}$-divisors.

Lemma 2.3. Let $L$ be a nef $\mathbb{R}$-divisor on a projective variety $X$. Then $L$ is big if and only if $L^{\operatorname{dim} X}>0$.

Proof. We put $n=\operatorname{dim} X$.

Step 1. If $L$ is big, then we can write $L \sim_{\mathbb{R}} A+D$ where $A$ is an ample $\mathbb{R}$-divisor and $D$ is an effective $\mathbb{R}$-Cartier divisor on $X$, where $\sim_{\mathbb{R}}$ denotes the $\mathbb{R}$-linear equivalence of $\mathbb{R}$-Cartier divisors. Then

$$
L^{n}=(A+D) \cdot L^{n-1} \geq A \cdot L^{n-1}=A \cdot(A+D) \cdot L^{n-2} \geq \cdots \geq A^{n}>0 .
$$

Hence $L^{\operatorname{dim} X}>0$ holds true when $L$ is big.

Step 2. In this step, we will check that $L$ is big under the assumption that $L^{n}>0$ holds. We will closely follow the proof of [La, Theorem 2.3.18]. We take ample $\mathbb{R}$-divisors $A_{1}$ and $A_{2}$ on $X$ such that $L+A_{1}$ and $A_{1}+A_{2}$ are $\mathbb{Q}$-Cartier divisors on $X$. Since $L^{n}>0$, we can assume that

$$
\left(L+A_{1}\right)^{n}>n\left(\left(L+A_{1}\right)^{n-1} \cdot\left(A_{1}+A_{2}\right)\right)
$$

holds by taking $A_{1}$ and $A_{2}$ sufficiently small. By the numerical criterion for bigness (see [La, Theorem 2.2.15]),

$$
L-A_{2}=\left(L+A_{1}\right)-\left(A_{1}+A_{2}\right)
$$

is big. Hence $L$ is also big.

We finish the proof of Lemma 2.3.

\section{Augmented base LOCi OF $\mathbb{R}$-Divisors}

In this section, we explain some properties of augmented base loci of $\mathbb{R}$-divisors following [B]. Let us start with the definition of base loci and stable base loci.

Definition 3.1 (Base loci and stable base loci of $\mathbb{Q}$-divisors). Let $X$ be a projective scheme and let $D$ be a Cartier divisor on $X$. The base locus of $D$ is defined as

$$
\mathrm{Bs}|D|=\left\{x \in X \mid \alpha \text { vanishes at } x \text { for every } \alpha \in H^{0}\left(X, \mathcal{O}_{X}(D)\right)\right\} .
$$

We consider $\operatorname{Bs}|D|$ with the reduced scheme structure.

The stable base locus of a $\mathbb{Q}$-Cartier divisor $L$ on $X$ is defined as

$$
\mathbf{B}(L)=\bigcap_{m} \operatorname{Bs}|m L|
$$

where $m$ runs over all positive integers such that $m L$ is Cartier. Note that $\mathbf{B}(L)$ is considered with the reduced scheme structure. 
The notion of augmented base loci plays a crucial role in the theory of minimal models.

Definition 3.2 (Augmented base loci of $\mathbb{R}$-divisors). Let $X$ be a projective scheme and let $L$ be an $\mathbb{R}$-Cartier divisor on $X$. We put

$$
\mathbf{B}_{+}(L)=\bigcap_{H} \mathbf{B}(L-H)
$$

where $H$ runs over all ample $\mathbb{R}$-divisors such that $L-H$ is $\mathbb{Q}$-Cartier. As usual, we consider $\mathbf{B}_{+}(L)$ with the reduced scheme structure. We call $\mathbf{B}_{+}(L)$ the augmented base locus of $L$.

Birkar defined $\mathbf{B}_{+}(L)$ differently (see [B, Definition 1,2]). Then he proved that his definition coincides with the usual one (see Definition 3.2). For the details, see [B, Lemma $3.1(3)]$.

In order to explain Birkar's theorem (see Theorem 3.4), it is convenient to introduce the notion of exceptional loci of $\mathbb{R}$-divisors.

Definition 3.3 (Exceptional loci of $\mathbb{R}$-divisors). Let $X$ be a projective scheme and let $L$ be an $\mathbb{R}$-Cartier divisor on $X$. The exceptional locus of $L$ is defined as

$$
\mathbb{E}(L)=\bigcup_{\operatorname{dim} V>0,\left.L\right|_{V} \text { is not big }} V,
$$

that is, the union runs over the positive-dimensional subvarieties $V \subset X$ such that $\left.L\right|_{V}$ is not big.

Note that $\mathbb{E}(L)$ is sometimes called the null locus of $L$ when $L$ is nef.

Theorem 3.4 ([B, Theorem 1.4]). Let $X$ be a projective scheme. Assume that $L$ is a nef $\mathbb{R}$-divisor on $X$. Then

$$
\mathbf{B}_{+}(L)=\mathbb{E}(L)
$$

holds.

For the details of Theorem 3.4, we strongly recommend the reader to see Birkar's original statement in [B, Theorem 1.4]. We will use Theorem 3.4 when $X$ is a normal projective variety in the proof of Theorem 1.4.

\section{Proof of Theorem 1.4}

In this section, we prove Theorem 1.4. The main ingredient of the proof of Theorem 1.4 below is Birkar's theorem (see Theorem 3.4).

Proof of Theorem 1.4. Let

$$
X=\bigcup_{i=1}^{k} U_{i}
$$

be a finite affine Zariski open cover of $X$. Let $\bar{U}_{i}$ be the closure of $U_{i}$ in $\mathbb{P}^{N_{i}}$. By [Lü, Lemma 2.2], which is an easy application of the flattening theorem (see [RG, Théorème (5.2.2)]), we can take an ideal sheaf $\mathcal{I}$ on $\bar{U}_{i}$ with $\operatorname{Supp} \mathcal{O}_{\bar{U}_{i}} / \mathcal{I} \subset \bar{U}_{i} \backslash U_{i}$ such that the blow-up of $\bar{U}_{i}$ along $\mathcal{I}$ eliminates the indeterminacy of $\bar{U}_{i} \rightarrow X$. Therefore, by taking the normalization of the blow-up of $\bar{U}_{i}$ along $\mathcal{I}$, we get a projective birational morphism $\pi_{i}: X_{i} \rightarrow X$ from a normal projective variety $X_{i}$ such that $\pi_{i}: \pi_{i}^{-1}\left(U_{i}\right) \rightarrow U_{i}$ is an isomorphism.

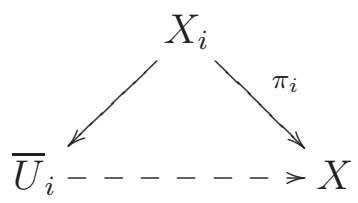


By Theorem 3.4, there exists an ample $\mathbb{R}$-divisor $H_{i}$ on $X_{i}$ such that $\pi_{i}^{*} L-H_{i}$ is $\mathbb{Q}$-Cartier and that

$$
\mathbf{B}\left(\pi_{i}^{*} L-H_{i}\right)=\mathbf{B}_{+}\left(\pi_{i}^{*} L\right)=\mathbb{E}\left(\pi_{i}^{*} L\right)
$$

holds. Let $\operatorname{Exc}\left(\pi_{i}\right)$ be the exceptional locus of $\pi_{i}$. Then

$$
\mathbb{E}\left(\pi_{i}^{*} L\right)=\operatorname{Exc}\left(\pi_{i}\right)
$$

holds by Lemma 2.3 and the assumption that $L^{\operatorname{dim} Z} \cdot Z>0$ for every positive-dimensional closed subvariety $Z \subset X$. Since $L$ is $\mathbb{R}$-Cartier, we can write

$$
L=\sum_{j \in J} l_{j} L_{j}
$$

such that $l_{j}$ is a real number and $L_{j}$ is Cartier for every $j \in J$. If $m_{j} \in \mathbb{Q}$ holds for every $j \in J$ and

$$
\pi_{i}^{*}\left(\sum_{j \in J} m_{j} L_{j}\right)-\pi_{i}^{*} L+H_{i}
$$

is ample, then

$$
\mathbf{B}\left(\pi_{i}^{*}\left(\sum_{j \in J} m_{j} L_{j}\right)\right) \subset \mathbf{B}\left(\pi_{i}^{*} L-H_{i}\right)=\operatorname{Exc}\left(\pi_{i}\right)
$$

holds. This implies that

$$
\mathbf{B}\left(\sum_{j \in J} m_{j} L_{j}\right) \subset \pi_{i}\left(\operatorname{Exc}\left(\pi_{i}\right)\right) \subset X \backslash U_{i} .
$$

Hence, there exists a positive real number $\varepsilon$ such that if $m_{j} \in \mathbb{Q}$ and $\left|l_{j}-m_{j}\right|<\varepsilon$ for every $j \in J$ then

$$
\mathbf{B}\left(\sum_{j \in J} m_{j} L_{j}\right) \subset \bigcap_{i=1}^{k}\left(X \backslash U_{i}\right)=\emptyset
$$

holds. This means that $\sum_{j \in J} m_{j} L_{j}$ is semi-ample. By this fact, we can write

$$
L=\sum_{p} r_{p} M_{p}
$$

such that $r_{p}$ is a positive real number and $M_{p}$ is a semi-ample $\mathbb{Q}$-divisor for every $p$. Therefore, $L$ is a semi-ample $\mathbb{R}$-divisor by definition. Thus there exist a morphism $f: X \rightarrow$ $Y$ onto a normal projective variety $Y$ with $f_{*} \mathcal{O}_{X} \simeq \mathcal{O}_{Y}$ and an ample $\mathbb{R}$-divisor $A$ on $Y$ such that $L$ is $\mathbb{R}$-linearly equivalent to $f^{*} A$ (see [F2, Lemma 2.1.11]). By assumption, $L \cdot C>0$ for every curve $C$ on $X$. This implies that $f$ is an isomorphism. Thus $L$ is an ample $\mathbb{R}$-divisor.

\section{Proof of Theorem 1.3}

In this section, we prove Theorem 1.3. More precisely, we reduce Theorem 1.3 to a special case where $X$ is a normal variety, which is nothing but Theorem 1.4. Let us start with the following elementary lemma.

Lemma 5.1. Let $X$ be a complete scheme and let $\mathcal{L}$ be an $\mathbb{R}$-line bundle on $X$. Let $X=\bigcup_{i=1}^{k} X_{i}$ be the irreducible decomposition of $X$. Then $\mathcal{L}$ is ample if and only if $\left.\mathcal{L}\right|_{\left(X_{i}\right)_{\mathrm{red}}}$ is ample for every $i$.

Proof. This statement is well known for $\mathbb{Q}$-line bundles. Hence we will freely use this lemma for $\mathbb{Q}$-line bundles in the following argument. 
Step 1. If $\mathcal{L}$ is ample, then it is obvious that so is $\left.\mathcal{L}\right|_{\left(X_{i}\right)_{\text {red }}}$ for every $i$. This is because we can write

$$
\mathcal{L}=\sum_{j} a_{j} \mathcal{L}_{j}
$$

where $\mathcal{L}_{j}$ is an ample line bundle on $X$ and $a_{j}>0$ for every $j$.

Step 2. In this step, we will prove that $\mathcal{L}$ is ample under the assumption that $\left.\mathcal{L}\right|_{\left(X_{i}\right)_{\text {red }}}$ is ample for every $i$. Since $\mathcal{L}$ is an $\mathbb{R}$-line bundle, we can write

$$
\mathcal{L}=\sum_{j=1}^{m} l_{j} \mathcal{L}_{j}
$$

where $\mathcal{L}_{j} \in \operatorname{Pic}(X)$ and $l_{j} \in \mathbb{R}$ for every $j$. We put

$$
V_{i}=\left\{\left(p_{1}, \ldots, p_{m}\right) \in \mathbb{R}^{m}\left|\sum_{j=1}^{m} p_{j} \mathcal{L}_{j}\right|_{\left(X_{i}\right)_{\text {red }}} \text { is ample }\right\}
$$

for every $i$. Then $V_{i}$ contains an open neighborhood of $l=\left(l_{1}, \ldots, l_{m}\right)$ for every $i$ since $\left.\sum_{j=1}^{m} l_{j} \mathcal{L}_{j}\right|_{\left(X_{i}\right)_{\text {red }}}$ is ample by assumption. Hence $V=\bigcap_{i=1}^{k} V_{i}$ contains a small open neighborhood of $l \in \mathbb{R}^{m}$. Thus we can take positive real numbers $r_{1}, \ldots, r_{p}$ and

$$
v_{1}=\left(v_{11}, \ldots, v_{1 m}\right), \ldots, v_{p}=\left(v_{p 1}, \ldots, v_{p m}\right) \in V \cap \mathbb{Q}^{m}
$$

such that $l=\sum_{\alpha=1}^{p} r_{\alpha} v_{\alpha}$. Then

$$
\mathcal{A}_{\alpha}:=\sum_{j=1}^{m} v_{\alpha j} \mathcal{L}_{j} \in \operatorname{Pic}(X) \otimes_{\mathbb{Z}} \mathbb{Q}
$$

is ample for every $\alpha$ since $v_{\alpha} \in V \cap \mathbb{Q}^{m}$. Since we can write

$$
\mathcal{L}=\sum_{\alpha=1}^{p} r_{\alpha} \mathcal{A}_{\alpha}
$$

$\mathcal{L}$ is ample by definition.

We finish the proof of Lemma 5.1.

Lemma 5.2. Let $X$ be a complete variety and let $\mathcal{L}$ be an $\mathbb{R}$-line bundle on $X$. Let $\pi: Y \rightarrow X$ be a finite surjective morphism between complete varieties. Then $\mathcal{L}$ is ample if and only if $\pi^{*} \mathcal{L}$ is ample.

Proof. This statement is well known for $\mathbb{Q}$-line bundles. Hence we will freely use this lemma for $\mathbb{Q}$-line bundles in this proof. Thus it is obvious that $\pi^{*} \mathcal{L}$ is ample when $\mathcal{L}$ is ample. Therefore, it is sufficient to prove that $\mathcal{L}$ is ample under the assumption that $\pi^{*} \mathcal{L}$ is ample. Since $\mathcal{L}$ is an $\mathbb{R}$-line bundle, we can write

$$
\mathcal{L}=\sum_{j=1}^{m} l_{j} \mathcal{L}_{j}
$$

where $\mathcal{L}_{j} \in \operatorname{Pic}(X)$ and $l_{j} \in \mathbb{R}$ for every $j$. Since $\pi^{*} \mathcal{L}$ is ample, there exists a positive real number $\varepsilon$ such that if $\left|l_{j}-\alpha_{j}\right|<\varepsilon$ for every $j$ then

$$
\pi^{*}\left(\sum_{j=1}^{m} \alpha_{j} \mathcal{L}_{j}\right)
$$

is ample. Moreover, if we further assume $\alpha_{j} \in \mathbb{Q}$ for every $j$, then

$$
\sum_{j=1}^{m} \alpha_{j} \mathcal{L}_{j}
$$


is ample since $\pi$ is a finite surjective morphism. Hence we can write

$$
\mathcal{L}=\sum_{i} r_{i} \mathcal{A}_{i}
$$

such that $r_{i}$ is a positive real number and $\mathcal{A}_{i}$ is an ample line bundle for every $i$. This means that $\mathcal{L}$ is ample by definition.

Let us prove Theorem 1.3.

Proof of Theorem 1.3. By Lemma 5.1, we may assume that $X$ is a variety. Let $\nu: X^{\nu} \rightarrow X$ be the normalization. Note that $\nu$ is a finite surjective morphism. Then by Lemma 5.2 it is sufficient to prove that $\nu^{*} \mathcal{L}$ is ample. Hence we may further assume that $X$ is a complete normal variety. In this case, the ampleness of $\mathcal{L}$ follows from Theorem 1.4.

\section{Proof of Theorem 1.5}

In this section, we prove Theorem 1.5. The following lemma is well known for $\mathbb{Q}$-line bundles.

Lemma 6.1. Let $\pi: X \rightarrow S$ be a proper surjective morphism between schemes and let $\mathcal{L}$ be an $\mathbb{R}$-line bundle on $X$. Assume that $\left.\mathcal{L}\right|_{X_{s}}$ is ample for every closed point $s \in S$, where $X_{s}=\pi^{-1}(s)$. Then $\mathcal{L}$ is $\pi$-ample, that is, we can write

$$
\mathcal{L}=\sum_{i} a_{i} \mathcal{L}_{i}
$$

in $\operatorname{Pic}(X) \otimes_{\mathbb{Z}} \mathbb{R}$ such that $\mathcal{L}_{i}$ is a $\pi$-ample line bundle on $X$ and $a_{i}$ is a positive real number for every $i$.

Before we prove Lemma 6.1, we prepare the following lemma, which is also well known for $\mathbb{Q}$-line bundles.

Lemma 6.2. Let $\pi: X \rightarrow S$ be a proper surjective morphism between schemes and let $\mathcal{L}$ be an $\mathbb{R}$-line bundle on $X$. Assume that $\left.\mathcal{L}\right|_{X_{s_{0}}}$ is ample for some closed point $s_{0} \in S$, where $X_{s_{0}}=\pi^{-1}\left(s_{0}\right)$. Then there exists a Zariski open neighborhood $U_{s_{0}}$ of $s_{0}$ such that $\left.\mathcal{L}\right|_{\pi^{-1}\left(U_{s_{0}}\right)}$ is ample over $U_{s_{0}}$.

Although Lemmas 6.1 and 6.2 are more or less known to the experts, we can not find them in the standard literature. Hence we prove them here for the sake of completeness.

Proof of Lemma 6.2. Since $\mathcal{L}$ is an $\mathbb{R}$-line bundle, there exist line bundles $\mathcal{M}_{j}$ for $1 \leq j \leq k$ such that

$$
\mathcal{L}=\sum_{j=1}^{k} b_{j} \mathcal{M}_{j}
$$

in $\operatorname{Pic}(X) \otimes_{\mathbb{Z}} \mathbb{R}$, where $b_{j} \in \mathbb{R}$ for every $j$. We put

$$
\mathcal{A}=\left\{\left(c_{1}, \ldots, c_{k}\right) \in \mathbb{R}^{k}\left|\sum_{j=1}^{k} c_{j} \mathcal{M}_{j}\right|_{X_{s_{0}}} \text { is ample }\right\} .
$$

Then $\mathcal{A}$ contains a small open neighborhood of $\left(b_{1}, \ldots, b_{k}\right)$. Hence we can write

$$
\mathcal{L}=\sum_{i} a_{i} \mathcal{L}_{i}
$$

where $\mathcal{L}_{i}$ is a line bundle on $X$ such that $a_{i}$ is a positive real number and $\left.\mathcal{L}_{i}\right|_{X_{s_{0}}}$ is ample for every $i$. Since $\left.\mathcal{L}_{i}\right|_{X_{s_{0}}}$ is ample for every $i$, there exists a Zariski open neighborhood $U_{s_{0}}$ of $s_{0}$ such that $\left.\mathcal{L}_{i}\right|_{\pi^{-1}\left(U_{s_{0}}\right)}$ is ample over $U_{s_{0}}$ for every $i$ (see, for example, [KoM, Proposition 1.41]). Therefore, $\left.\mathcal{L}\right|_{\pi^{-1}\left(U_{s_{0}}\right)}=\left.\sum_{i} a_{i} \mathcal{L}_{i}\right|_{\pi^{-1}\left(U_{s_{0}}\right)}$ is ample over $U_{s_{0}}$. 
Let us prove Lemma 6.1.

Proof of Lemma 6.1. We use the same notation as in the proof of Lemma 6.2. By Lemma 6.2 , we can take $s_{1}, \ldots, s_{l} \in S$ such that

$$
\bigcup_{\alpha=1}^{l} U_{s_{\alpha}}=S
$$

and that $\left.\mathcal{L}\right|_{\pi^{-1}\left(U_{s_{\alpha}}\right)}$ is ample over $U_{s_{\alpha}}$ for every $\alpha$. We put

$$
\mathcal{A}_{\alpha}=\left\{\left(c_{1}, \ldots, c_{k}\right) \in \mathbb{R}^{k}\left|\sum_{j=1}^{k} c_{j} \mathcal{M}_{j}\right|_{\pi^{-1}\left(U_{s_{\alpha}}\right)} \text { is } \pi \text {-ample over } U_{s_{\alpha}}\right\} .
$$

Then $\mathcal{A}_{\alpha}$ contains a small open neighborhood of $\left(b_{1}, \ldots, b_{k}\right)$. Therefore, $\bigcap_{\alpha=1}^{l} \mathcal{A}_{\alpha}$ also contains a small open neighborhood of $\left(b_{1}, \ldots, b_{k}\right)$. Hence we can write

$$
\mathcal{L}=\sum_{i} a_{i} \mathcal{L}_{i}
$$

in $\operatorname{Pic}(X) \otimes_{\mathbb{Z}} \mathbb{R}$ such that $\mathcal{L}_{i}$ is a $\pi$-ample line bundle on $X$ and $a_{i}$ is a positive real number for every $i$.

Finally, we prove Theorem 1.5.

Proof of Theorem 1.5. If $\mathcal{L}$ is $\pi$-ample, then it is obvious that it satisfies the desired property. Hence, by Lemma 6.1, it is sufficient to prove that $\left.\mathcal{L}\right|_{X_{s}}$ is ample for every closed point $s \in S$, where $X_{s}=\pi^{-1}(s)$, under the assumption that $\mathcal{L}^{\operatorname{dim} Z} \cdot Z>0$. This follows from the Nakai-Moishezon ampleness criterion for $\mathbb{R}$-line bundles on complete schemes (see Theorem 1.3).

\section{Proof of TheOrem 1.6}

In this final section, we prove the Nakai-Moishezon ampleness criterion for $\mathbb{R}$-line bundles on complete algebraic spaces (Theorem 1.6) by using some basic properties of algebraic spaces and Theorem 1.3.

Proof of Theorem 1.6. It is well known that the Nakai-Moishezon ampleness criterion holds for line bundles on complete algebraic spaces (see, for example, [Ko, 3.11. Theorem] and [P, (1.4) Theorem]). It is also well known that there exists a finite surjective morphism $f: Y \rightarrow X$ from a complete scheme $Y$ (see, for example, [Ko, 2.8. Lemma]). By Theorem $1.3, f^{*} \mathcal{L}$ is an ample $\mathbb{R}$-line bundle on $Y$. We write

$$
\mathcal{L}=\sum_{j} a_{j} \mathcal{L}_{j}
$$

where $\mathcal{L}_{j}$ is a line bundle on $X$ and $a_{j}$ is a real number for every $j$. We put

$$
\mathcal{M}=\sum_{j} b_{j} \mathcal{L}_{j}
$$

where $b_{j}$ is a rational number for every $j$. If $\left|a_{j}-b_{j}\right| \ll 1$ for every $j$, then $f^{*} \mathcal{M}$ is an ample $\mathbb{Q}$-line bundle on $Y$ since $f^{*} \mathcal{L}$ is ample. Therefore, $m \mathcal{M}$ is an ample line bundle on $X$ for some positive integer $m$ by the Nakai-Moishezon ampleness criterion for line bundles on complete algebraic spaces. This implies that $X$ is projective. Thus, by Theorem 1.3 again, $\mathcal{L}$ is an ample $\mathbb{R}$-line bundle on $X$. 


\section{REFERENCES}

[B] C. Birkar, The augmented base locus of real divisors over arbitrary fields, Math. Ann. 368 (2017), no. 3-4, 905-921.

[CP] F. Campana, T. Peternell, Algebraicity of the ample cone of projective varieties, J. Reine Angew. Math. 407 (1990), 160-166.

[F1] O. Fujino, On the Kleiman-Mori cone, Proc. Japan Acad. Ser. A Math. Sci. 81 (2005), no. 5, 80-84.

[F2] O. Fujino, Foundations of the minimal model program, MSJ Memoirs, 35. Mathematical Society of Japan, Tokyo, 2017.

[F3] O. Fujino, Semipositivity theorems for moduli problems, Ann. of Math. (2) 187 (2018), no. 3, 639-665.

[F4] O. Fujino, Minimal model theory for $\log$ surfaces in Fujiki's class $\mathcal{C}$, to appear in Nagoya Math. J.

[FP] O. Fujino, S. Payne, Smooth complete toric threefolds with no nontrivial nef line bundles, Proc. Japan Acad. Ser. A Math. Sci. 81 (2005), no. 10, 174-179.

[Kl] S. L. Kleiman, Toward a numerical theory of ampleness, Ann. of Math. (2) 84 (1966), 293-344.

[Ko] J. Kollár, Projectivity of complete moduli, J. Differential Geom. 32 (1990), no. 1, 235-268.

$[\mathrm{KoM}]$ J. Kollár, S. Mori, Birational geometry of algebraic varieties. With the collaboration of C. H. Clemens and A. Corti. Translated from the 1998 Japanese original. Cambridge Tracts in Mathematics, 134. Cambridge University Press, Cambridge, 1998.

[La] R. Lazarsfeld, Positivity in algebraic geometry. I. Classical setting: line bundles and linear series, Ergebnisse der Mathematik und ihrer Grenzgebiete. 3. Folge. A Series of Modern Surveys in Mathematics [Results in Mathematics and Related Areas. 3rd Series. A Series of Modern Surveys in Mathematics], 48. Springer-Verlag, Berlin, 2004.

[Lü] W. Lütkebohmert, On compactification of schemes, Manuscripta Math. 80 (1993), no. 1, 95-111.

[P] P. Pascual Gainza, Ampleness criteria for algebraic spaces, Arch. Math. (Basel) 45 (1985), no. 3, 270-274.

[RG] M. Raynaud, L. Gruson, Critères de platitude et de projectivitè. Techniques de "platification" d'un module, Invent. Math. 13 (1971), 1-89.

Osamu Fujino, Department of Mathematics, Graduate School of Science, Osaka UniVERSiTy, TOYONAKA, OSAKa 560-0043, JAPAN

Email address: fujino@math.sci.osaka-u.ac.jp

Keisuke Miyamoto, Department of Mathematics, Graduate School of Science, Osaka University, TOYONAKA, OSAKA 560-0043, JAPAN

Email address: u901548b@ecs.osaka-u.ac.jp 